\title{
APLICACIÓN DE BIOENSAYOS EN LA MEDICIÓN DE TOXICIDAD POR METALES PESADOS EN FUENTES SUPERFICIALES DE AGUA PARA CONSUMO HUMANO
}

\author{
Responsable : MSc. César Julio Cáceda Quiroz \\ Miembro : Mblgo, Anacelly Valera Lopez
}

\begin{abstract}
RESUMEN
Se evaluó el efecto tóxico potencial de metales pesados de una muestra de agua del rio Santa sobre el crecimiento de la raiz de Allium cepa y Lactuca sativa. De acuerdo con los resultados obtenidos la aplicación de bioensayos con Allium cepa fue más sensible a la presencia de metales de plomo y cromo con respecto a Lactuca sativa.
\end{abstract}

\section{ABSTRACT}

It evalued the potential toxic effect of heavy metals a sample water from Santa river, about the root growth Allium cepa and Lactuca sativa. In accordance with obtained results the biotesting application with Allium cepa, it was more sensible to the lead and chrome metals presence with respect to the Lactuca sativa.

\section{INTRODUCCIÓN}

Muchos paises de América Latina cuentan con reglamentaciones sobre protección de los recursos naturales, como el agua. Se ha puesto énfasis en la contaminación orgánica y de organismos patógenos, mientras que la problemática de la descarga de compuestos tóxicos y manejo de residuos peligrosos recién comienza a ser considerada.. Una de las causas de esta falencia se relaciona con la falta de infraestructura y experiencias necesarias para la detección de contaminantes tóxicos complejos que, de una u otra forma, generan riesgos para la salud humana y los ecosistemas naturales.

Un agua clara y potable es una necesidad humana básica, pero el acceso a ella continúa siendo una gran dificultad para algunas ciudades en paises en desarrollo como el nuestro. En la actualidad se reconoce que la caracterización y medición de los tóxicos o componentes de los residuos peligrosos por separado no es suficiente para asegurar la ausencia de efectos indeseables, puesto que tanto la mezcla de los residuos como posibles transformaciones en el ambiente pueden modificar su efecto nocivo. De ahí que el uso de ensayos biológicos está siendo considerado cada vez con mayor intensidad para la evaluación de la toxicidad global de estos contaminantes

El propósito del trabajo fue poner en consideración de los profesionales que trabajan en el control de calidad, área de laboratorio de microbiologia de las EPS la aplicación de bioensayos que permitan evaluar el efecto tóxico potencial de los contaminantes y sea considerado una prueba de alerta en los programas de monitoreo en la calidad de las fuentes de agua, teniendo en cuenta la implementación de un equipamiento básico con insumos mínimos.

\section{OBJETIVO}

Evaluar el efecto tóxico potencial de metales pesados de muestras de aguas del rio Santa sobre el crecimiento de la raiz de Allium cepa y Lactuca sativa.

\section{MATERIALYMÉTODOS}

1) Materiales:

Muestra de agua del rio Santa.Bulbos de Allium cepa, de aprox. $2,5 \mathrm{~cm}$ de diámetro, secos y $\sin$ formación de hojas y/o raiz. Medio de crecimiento, de pH 7,0. Semillas de Lactuca sativa. Agua dura reconstituida (APHA, 1992). Los de uso común en laboratorio.

2) Ensayo de Toxicidad Aguda con Allium cepa mediante la Evaluación de la Inhibición del Crecimiento Promedio de Raices de Cebolla.

Se utilizaron bulbos de $2,5 \mathrm{~cm}$ de diámetro, los que fueron adquiridos 3 dias antes de la realización de las pruebas y almacenados en un ambiente seco.

Se empleó una serie de 5 concentraciones de la muestra y un control negativo, cada uno con doce réplicas el cual permitió establecer el intervalo de concentración conveniente para la determinación inhibitoria media $\left(\mathrm{Cl}_{50}\right)$. El ensayo se inició con el llenado de los vasos con cada una de las muestras de agua. Se colocaron los bulbos limpios sobre la boca de los vasos, cuidando que la zona radicular quede inmersa en el liquido. Las muestras se colocaron sobre una mesa estable que no presentase vibraciones y se mantuvieron a una temperatura ambiente $\left(20^{\circ} \mathrm{C}\right)$ por un periodo de 72 horas, evitándose la iluminación directa. Al término del periodo de exposición se registró la longitud promedio de las raices, con ayuda de una regla. Se realizó la siguiente operación: (ongitud del control longitud de la muestra) x 100/longitud del control). Con estos valores se construyó una gráfica de concentración en función del porcentaje de inhibición y se calculó la $\mathrm{Cl}_{50}$. 


\section{3) Ensayo de la Toxicidad Aguda con Semillas de Lactuca sativa.}

Es una prueba estática de toxicidad aguda (120 horas de exposición) en la que se evalúan los efectos citotóxicos de compuestos puros o de mezclas complejas en el proceso de germinación de las semillas y en el desarrollo de plántulas durante los primeros días de crecimiento.

Las semillas de $L$. sativa certificadas fueron adquiridas en una semilleria local, las cuales se almacenaron $14^{\circ} \mathrm{C}$, en oscuridad y en ambiente seco.

Para la realización de la curva dosis-respuesta se prepararon cinco diluciones de la muestra y un control negativo, realizándose 5 repeticiones para cada dilución.

Se colocó en cada placa Petri un disco de papel de filtro. Se saturó el papel de filtro con 4 ó $5 \mathrm{~mL}$ de la dilución, evitando la formación de bolsas de aire. Con una pinza se colocarón cuidadosamente 20 semillas, dejando espacio suficiente entre ellas para permitir la elongación de las raíces.

Se taparon las placas y se colocaron en bolsas plásticas para evitar la pérdida de humedad. Se incubó durante 120 horas (5 dias) a una temperatura de $22 \pm 2$ ${ }^{\circ} \mathrm{C}$.

Terminado el periodo de exposición (120 horas), se procedió a cuantificar el efecto de la germinación y en la elongación de la radicula y del hipocótilo.

Se registró el número de semillas que germinaron normalmente, teniendo como criterio de germinación la aparición visible de la radicula.

Utilizando una regla o papel milimetrado se midió cuidadosamente la longitud de la radicula y del hipocótilo de cada una de las plántulas correspondientes a cada concentración de tóxico o dilución de muestra y a los controles. La medida de elongación de la radicula se consideró desde el nudo hasta el ápice radicular. La medida de elongación del hipocótilo se consideró desde el nudo hasta el sitio de inserción de los dos cotiledones.

Los resultados se expresaron de acuerdo con los siguientes cálculos:

- Promedio de la elongación de la radicula y del hipocótilo de las plántulas de cada repetición.

- Porcentaje de inhibición del crecimiento de la radicula y del hipocótilo, con el promedio de elongación para cada dilución respecto del promedio de elongación del control negativo.

- Porcentaje de inhibición en la germinación.

- Cálculo del gráfico dosis respuesta y $\mathrm{Ci}_{50}$.

\section{RESULTADOS}

Cuadro $N^{0}$ 01: Resultados de la prueba de toxicidad del plomo utilizando Allium cepa.

\begin{tabular}{|c|c|c|c|c|c|c|c|c|c|c|}
\hline \multirow[b]{2}{*}{ NUESTRA } & \multirow{2}{*}{$\begin{array}{c}\text { CONCENTRACION } \\
\text { agl }\end{array}$} & \multicolumn{5}{|c|}{ Longhud of a raizen om } & \multirow[b]{2}{*}{ is } & \multirow[b]{2}{*}{ PROUEDIO } & \multirow[b]{2}{*}{$\begin{array}{c}x \\
\text { WhEBicion }\end{array}$} & \multirow[b]{2}{*}{$\begin{array}{l}\text { MaCE } \\
\text { urTONCC }\end{array}$} \\
\hline & & Réplica & $\begin{array}{c}\text { Rieplica } \\
2\end{array}$ & Replica & Riplica & $\begin{array}{c}\text { Regplita } \\
5\end{array}$ & & & & \\
\hline $\begin{array}{l}\text { Control } \\
\text { Negativo }\end{array}$ & 0,00 & 3,8 & 2,5 & 2,9 & 2,7 & 3.3 & 1,32 & 3,04 & 0.00 & 100 \\
\hline 1 & 0.01 & 1,8 & 1,4 & 1,6 & 2,0 & 1,8 & 0,73 & 1,72 & 43,42 & 76 \\
\hline 2 & 0,06 & 0,8 & 0,6 & 0,8 & 0,9 & 0,9 & 0,32 & 0,80 & 73.68 & 42 \\
\hline 3 & 0,11 & 0,4 & 0,5 & 0.2 & 0,6 & 0,4 & 0.18 & 0,42 & 86,18 & 29 \\
\hline 4 & 0,16 & 0,3 & 0,0 & 0.2 & 0,3 & 0,2 & 0,11 & 0.20 & 93,42 & 18 \\
\hline 5 & 0.21 & 0,1 & 0,0 & 0.2 & 0,0 & 0,1 & 0,09 & 0.08 & 97,37 & 5 \\
\hline
\end{tabular}

Cuadro 02: Resultados de la Prueba de Toxicidad del Cromo Utilizando Allium Cepa

\begin{tabular}{|c|c|c|c|c|c|c|c|c|c|c|}
\hline \multirow{2}{*}{ MUESTRA } & \multirow{2}{*}{$\begin{array}{c}\text { CONCEnTRACIOM } \\
\mathrm{mgl}\end{array}$} & \multicolumn{5}{|c|}{ Lengitsd de la rize en em } & \multirow[b]{2}{*}{ os } & \multirow[b]{2}{*}{ PROWEDIO } & \multirow{2}{*}{${ }_{\text {NHBBCON }}^{5}$} & \multirow{2}{*}{$\begin{array}{l}\text { INOCE } \\
\text { WTOTCOCO }\end{array}$} \\
\hline & & $\begin{array}{c}\text { Réplica } \\
1\end{array}$ & $\begin{array}{c}\text { Riplica } \\
2\end{array}$ & $\frac{\text { Répica }}{3}$ & Riplia & $\underset{5}{R e p l i t a}$ & & & & \\
\hline $\begin{array}{l}\text { Control } \\
\text { Negativo }\end{array}$ & 0,0 & 5,6 & 3,4 & 4,3 & 5,2 & 4,9 & 2,06 & 4,68 & 0,00 & 150 \\
\hline 1 & 0,5 & 4,2 & 4,2 & 5,1 & 4,5 & 4,1 & 1,64 & 4,42 & 5,56 & 150 \\
\hline 2 & 1,0 & 3,3 & 3,0 & 3.6 & 3.5 & 3.2 & 0,97 & 3,32 & 29,06 & 98 \\
\hline 3 & 1,5 & 3,1 & 2,5 & 2,2 & 2.9 & 2.5 & 0,56 & 2,64 & 43,59 & 97 \\
\hline 4 & 2,0 & 2,0 & 2,2 & 2.5 & 2,0 & 2,0 & 0,20 & 2,14 & 54,27 & 82 \\
\hline 5 & 2,5 & 2,0 & 1,9 & 2,1 & 1,7 & 2,2 & 0,27 & 1,98 & 57,69 & 70 \\
\hline
\end{tabular}

Cuadro 03: Resultados de la Prueba de Toxicidad del Plomo Utilizando Lactuca Sativa Inhibicion de la Germinaciçon

\begin{tabular}{|c|c|c|c|c|}
\hline MUESTRA & $\begin{array}{c}\text { CONCENTRACION } \\
\text { mgII }\end{array}$ & $\begin{array}{c}\text { N PROMEDIO DE } \\
\text { SEMLLAS GERMINADAS }\end{array}$ & $\begin{array}{c}\text { \% SEMILLAS } \\
\text { GERMINADAS }\end{array}$ & $\begin{array}{c}\text { \% SEMILLAS } \\
\text { GERMIINAC. INHIIBDA }\end{array}$ \\
\hline Control Negatio & 0,00 & 20 & 100 & 0 \\
\hline 1 & 0,01 & 20 & 100 & 0 \\
\hline 2 & 0,06 & 20 & 100 & 0 \\
\hline 3 & 0,11 & 20 & 100 & 0 \\
\hline 4 & 0,16 & 18 & 90 & 10 \\
\hline 5 & 0,21 & 15 & 75 & 25 \\
\hline
\end{tabular}

Cuadro $\mathrm{N}^{\circ}$ 04: Resultado de la prueba de toxicidad del plomo utilizando lactuca siva Inhibición de la germinación

\begin{tabular}{|c|c|c|c|c|}
\hline MUESTRA & $\begin{array}{c}\text { CONCENTRACIOH } \\
\mathrm{mgl}\end{array}$ & $\begin{array}{c}\text { NP PROMEDIO DE } \\
\text { SEMLLAS GERMIINADAS }\end{array}$ & $\begin{array}{l}\text { \% SEMILLAS } \\
\text { GERMIMADAS }\end{array}$ & $\begin{array}{c}\text { Y SEEMLLAS } \\
\text { GERMINAC. WHIBDA }\end{array}$ \\
\hline Control Negativo & 0 & 20 & 100 & 0 \\
\hline 1 & 0,5 & 20 & 100 & 0 \\
\hline 2 & 1 & 19 & 95 & 5 \\
\hline 3 & 1,5 & 17 & 85 & 15 \\
\hline 4 & 2 & 15 & 75 & 25 \\
\hline 5 & 2,5 & 13 & 65 & 35 \\
\hline
\end{tabular}


Cuadro $N^{0}$ 5: Resultados de la prueba de toxicidad del plomo utilizando lactuca sativa Inhibición del crecimiento de la raiz

\begin{tabular}{|c|c|c|c|c|}
\hline MUESTRA & $\begin{array}{c}\text { CONCENTRACION } \\
\text { mgll }\end{array}$ & $\begin{array}{c}\text { LONGIUD EN CM DE } \\
\text { LARAZ (PROMEDIO) }\end{array}$ & $\begin{array}{c}\text { \% } \\
\text { CRECIMIENTO }\end{array}$ & $\begin{array}{c}\% \\
\text { INHIBCION }\end{array}$ \\
\hline Control Negativo & 0,00 & 2,9 & 100,00 & 0,00 \\
\hline 1 & 0,01 & 2,6 & 89,66 & 10,34 \\
\hline 2 & 0,06 & 2,1 & 72,41 & 27,59 \\
\hline 3 & 0,11 & 1,9 & 65,52 & 34,48 \\
\hline 4 & 0,16 & 1,8 & 62,07 & 37,93 \\
\hline 5 & 0,21 & 1,6 & 55,17 & 44,83 \\
\hline
\end{tabular}

Cuadro $N^{\circ}$ 6: Resultados de la prueba de toxicidad del cromo utilizando lactuca sativa Inhibición del crecimiento de la raiz

\begin{tabular}{|c|c|c|c|c|}
\hline MUESTRA & $\begin{array}{c}\text { CONCENTRACION } \\
\text { mg/ }\end{array}$ & $\begin{array}{c}\text { LONGITUD EN CM DE } \\
\text { LARAIZ (PROMEDIO) }\end{array}$ & $\begin{array}{c}\% \\
\text { CRECIMIENTO }\end{array}$ & $\begin{array}{c}\% \\
\text { INHIBICION }\end{array}$ \\
\hline Control Negativo & 0,0 & 2,9 & 100,00 & 0,00 \\
\hline 1 & 0,5 & 2,6 & 89,66 & 10,34 \\
\hline 2 & 1,0 & 2,1 & 72,41 & 27,59 \\
\hline 3 & 1,5 & 1,9 & 65,52 & 34,48 \\
\hline 4 & 2,0 & 1,8 & 62,07 & 37,93 \\
\hline 5 & 2,5 & 1,6 & 55,17 & 44,83 \\
\hline
\end{tabular}

\section{DISCUSIÓN}

De acuerdo con los resultados obtenidos, la aplicación de bioensayos con Allium cepa fue más sensible a la presencia de metales de plomo $(\mathrm{Pb})$ y cromo $(\mathrm{Cr})$ con respecto a Lactuca sativa, esto se demuestra en los resultados obtenidos. En las pruebas con $A$. cepa con respecto al $\mathrm{Pb}$, el porcentaje de inhibición de las raíces se obtuvo a una concentración de $0,21 \mathrm{mg} / \mathrm{L}$, mientras que con $\mathrm{Cr}$ se obtuvo a una concentración de $2,5 \mathrm{mg} / \mathrm{L}$, esto nos indica que a concentraciones menores de $\mathrm{Pb}$ existe un menor crecimiento de la raíz en $\boldsymbol{A}$. Cepa.

Es decir, que su efecto es más letal a concentraciones bajas (ver cuadros 1 y 2). Así mismo el menor índice mitótico se obtuvo en presencia de $\mathrm{Pb}$.

Si aplicamos estos resultados con los valores de los puntos de muestreo a lo largo del río Santa, nos damos cuenta de que en 8 de estos puntos existen valores por encima de $0,1 \mathrm{mg} / \mathrm{L}$, lo que indica la presencia de este metal con efectos ecotóxicos, lo que no sucede con el cromo, ya que en condiciones normales de los valores de este último metal a lo largo del río Santa no representa un riesgo toxicológico; por lo tanto, no representa un riesgo a la salud. Con respecto a $L$. sativa en las pruebas de inhibición de la germinación (ver cuadros 3 y 4 ) tanto el $\mathrm{Cr}$ como el $\mathrm{Pb}$ a las condiciones trabajadas no afectan significativamente la germinación de $L$. sativa. Así mismo la concentración que inhibió al $50 \%$ el crecimiento de la raiz se obtuvo entre 0,16 a $0,21 \mathrm{mg} / \mathrm{L}$ en el caso del $\mathrm{Pb}$, mientras que con el $\mathrm{Cr}$ se necesita valores mayores a $2,5 \mathrm{mg} / \mathrm{L}$ para poder inhibir el crecimiento de la raiz a un $50 \%$.

Los resultados obtenidos en este trabajo deben ser una voz de alerta para las autoridades e instituciones que vigilan la calidad del río Santa, ya que esta considerada como fuente o rio de clase II, es decir fuente para abastecimiento de agua para consumo humano y el riego de especies agrícolas de consumo humano, de tal manera que se puedan controlar estos valores y que no representen niveles de toxicidad que afecten la salud humana, ya que si estos valores persisten en el agua de consumo humano una vez realizado el tratamiento seria necesario plantear posibilidades de buscar otras fuentes para este propósito. Estos resultados a pesar de ser preliminares y quizás insuficientes, son una buena base para conocer los niveles de toxicidad presentes a lo largo del recorrido del rio Santa. Con un muestreo en campo y más amplio de la zona se podrian llevar estos resultados para establecer un seguimiento de los puntos de vertido y de las medidas que se deben tomar para controlar las fuentes de contaminación.

\section{CONCLUSIONES}

1. El cromo en concentraciones mayores a $1,5 \mathrm{mg} / \mathrm{L}$ inhibe el $50 \%$ el crecimiento de la raiz $\left(\mathrm{IC}_{50}\right)$, y a concentraciones mayores de $2,5 \%$ disminuye las mitosis de las células meristemáticas de A. cepa.

2. El plomo en concentraciones mayores a $0,06 \mathrm{mg} / \mathrm{L}$ inhibe en $50 \%\left(\mathrm{IC}_{50}\right)$ el crecimiento de la raiz y el $50 \%$ de las mitosis de las células meristemáticas de $A$. cepa.

3. El $\mathrm{Cr}$ y el $\mathrm{Pb}$, a las concentraciones trabajadas no influyen en la germinación de L. sativa.

4. El plomo es el metal más sensible en los bioensayos trabajados.

5. La aplicación de bioensayos son útiles como una prueba de alerta en los programas de monitoreo de la calidad de las fuentes de agua.

\section{BIBLIOGRAFÍA}

Aquatox". Mayo 2003". Aquatox: juventud, ciencia, salud y medio ambiente". Centro Internacional de Investigaciones para el Desarrollo (CIID/IDRC), de Canadá. Programa "Enfoques Ecosistémicos para la Salud (Ecosalud).

APHA, 1998. Standard Methodsfor the Examination of Water and Wastewater, 20th ed., American Public Health Association, Washington, D.C.

Aurazo Margarita.2003. Bioindicadores Biológicos de calidad de Fuentes y bioensayos de toxicidad. Trujillo-Perú.

Butler, G.C., 1978, Principles of Ecotoxicology, SCOPE 12, John Wiley and Sons, New York.

Cheung, Y. H., M. H., Wong y N. F. Y., Tam. 1989. "Root and Shoot Elongation as an Assessment of Heavy Metal Toxicity" and "Zn Equivalent Value' of Edible Crops", Hydrobiologia

Díaz-Báez, M.C., Cruz, L.E., Rodríguez, D., Pérez, J. \& Vargas, C.M., 2000, "Evaluation of Three Water Concentration Techniques as a Prior Step to Test Acute Toxicity", Environ. Toxicol.

Dutka, B.J., 1996, Bioassays: a Historical Summary of those Used and Developed in our Laboratories at NWRI. National Water Research Institute, Environment Canada, Burlington 
Fiskesjö, G. 1997. "Allium Test for Screening Chemicals; Evaluation of Cytological Parameters", in: Plants for Environmental Studies, Wancheng, W.; J. W. Gorsuch, J. S. Hughes eds., CRC Press, Florida.

Instituto Mexicano de Tecnología del agua. 2004 "Ensayos toxicológicos y Métodos de Evaluación de la calidad del agua". México

IB- Red Interinstitucional de Bioensayos 2002. Mayo a Setiembre de 2002. "Los bioensayos como herramienta de evaluación de la toxicidad de los efluentes industriales en Uruguay". Trabajo financiado por el CIID/IDRC de Canadá, conjuntamente con DINAMA, LATU, IMM y F. CIENCIAS.

OMS. 1985. "Guia para la calidad del agua potable. Washington. EUA.

Rojas, Ricardo. 2003. "Vigilancia y control de la calidad del agua para consumo humano".Lima.

Steel, R.G.D. y Torrie, J.H., 1985, Bioestadistica, principios y procedimientos, 2da ed., Editorial McGraw Hill, Bogotá.

UNAM. 1986. Biología celular: Manual de prácticas. Departamento de Biologia, Facultad de Ciencias, Universidad Nacional Autónoma de México. 